\title{
First-principles analysis of lattice thermal conductivity in monolayer and bilayer graphene
}

\author{
B. D. Kong, ${ }^{1}$ S. Paul, ${ }^{2}$ M. Buongiorno Nardelli, ${ }^{2,3}$ and K. W. Kim ${ }^{1, *}$ \\ ${ }^{1}$ Department of Electrical and Computer Engineering, North Carolina State University, Raleigh, North Carolina 27695-7911, USA \\ ${ }^{2}$ Department of Physics, North Carolina State University, Raleigh, North Carolina 27695-8202, USA \\ ${ }^{3}$ Computer Science and Mathematics Division, Oak Ridge National Laboratory, Oak Ridge, Tennessee 37831, USA
}

(Received 23 February 2009; revised manuscript received 5 June 2009; published 9 July 2009)

\begin{abstract}
Using calculations from first principles, we investigate the lattice thermal conductivity of ideal monolayer and bilayer graphenes. Our result estimates that the intrinsic thermal conductivity of both materials is around $2200 \mathrm{~W} \mathrm{~m}^{-1} \mathrm{~K}^{-1}$ at $300 \mathrm{~K}$, a value close to the one observed theoretically and experimentally in graphite along the basal plane. It also illustrates the expected $T^{-1}$ dependence at higher temperatures. The little variation between monolayer and bilayer thermal conductivities suggests that the number of layers may not affect significantly the in-plane thermal properties of these systems. The intrinsic thermal conductivity also appears to be nearly isotropic for graphene.
\end{abstract}

DOI: 10.1103/PhysRevB.80.033406

PACS number(s): 68.65.-k, 65.80.+n, 81.05.Uw, 63.20.-e

Although carbon-based low-dimensional materials have been a subject of intense investigations for decades, the recent synthesis of graphene, a single two-dimensional (2D) sheet of carbon atoms in a honeycomb lattice, has opened a unique avenue of research in the search for novel alternative materials for microelectronics. ${ }^{1}$ The peculiar electronic structure of graphene ${ }^{1}$ implies that electrons have a negligible effective mass around the $K$ point of the Brillouin zone (Dirac point) and thus a high electrical conductivity. Subsequent anticipation of the superior performance in device applications also makes it imperative to investigate the thermal properties of these systems as electric currents are inherently coupled with Joule heating. Usually a good electric conductor is a good thermal conductor. However, this expectation does not apply in the case of diamond, a material made of carbon atoms, which exhibits a high thermal conductivity despite its large band gap and low electric conductivity. Graphite, another carbon-based material and the closest relative of graphene, displays a high thermal conductivity along the basal plane while that in the $c$ axis is much smaller. ${ }^{2}$

Available experimental data show a relatively broad range for the thermal conductivity of highly oriented graphite and graphene systems. The values of $1660-1880 \mathrm{~W} \mathrm{~m}^{-1} \mathrm{~K}^{-1}$ were cited for graphite in Ref. 2 while those measured in a suspended sheet of monolayer graphene were around $4840-5300 \mathrm{~W} \mathrm{~m}^{-1} \mathrm{~K}^{-1}$ to $3080-5150 \mathrm{~W} \mathrm{~m}^{-1} \mathrm{~K}^{-1} \cdot 3,4$ Very recently, Nika et al. ${ }^{5}$ reported calculated results for monolayer graphene between 2000-6000 $\mathrm{W} \mathrm{m}^{-1} \mathrm{~K}^{-1}$, depending on the choice of Grüneisen parameter $\gamma$ (varying from 0.8 to 2.0) as deduced from Ref. 6. The latter study was based on a valence-force-field method (which approximates all interatomic forces to bond-bending and stretching modes). ${ }^{7}$ Complementing the earlier investigations, the purpose of the present Brief Report is to theoretically examine the characteristic thermal response of monolayer and bilayer graphenes by taking advantage of the first-principles approach. The results clearly indicate the superior intrinsic thermal conductivity of this unique material when compared to the conventional bulk solids including diamond $\left(600-2000 \mathrm{~W} \mathrm{~m}^{-1} \mathrm{~K}^{-1}\right){ }^{8}$

In general, the lattice thermal conductivity $\kappa$ of a crystal at finite temperature $T$ can be written as $\kappa=\Sigma_{\lambda} \kappa_{\lambda}$

$$
\kappa_{\lambda}=\sum_{\mathbf{q}}\left[\mathbf{v}_{\lambda}(\mathbf{q}) \cdot \mathbf{t}\right]^{2} \tau_{\lambda}(\mathbf{q}) C_{p h}\left(\omega_{\lambda}\right),
$$

where $\mathbf{t}$ is a unit vector in the direction of thermal gradient $\nabla T, \mathbf{v}(\mathbf{q})$ is the group velocity of a phonon with wave vector $\mathbf{q}$, and $\tau(\mathbf{q})$ is its lifetime. ${ }^{9-11}$ The index $\lambda$ runs over the phonon modes and $C_{p h}(\omega)$ is the contribution of phonon modes to the specific heat,

$$
C_{p h}(\omega)=\hbar \omega \frac{d N^{0}}{d T}=\frac{(\hbar \omega)^{2}}{k_{B} T^{2}} \frac{\exp \left(\hbar \omega / k_{B} T\right)}{\left[\exp \left(\hbar \omega / k_{B} T\right)-1\right]^{2}},
$$

where $k_{B}$ is the Boltzmann constant and $\omega$ is the phonon frequency. Accordingly, a detailed knowledge of phonon dynamics is essential to accurately evaluate the lattice thermal conductivity [specifically, the energy $\omega_{\lambda}(\mathbf{q})$, the group velocity $\mathbf{v}_{\lambda}(\mathbf{q})$, and the lifetime $\tau_{\lambda}(\mathbf{q})$ for all $\mathbf{q}$ and $\left.\lambda\right]$. This can be provided by the first-principles calculations within the density-functional theory (DFT) and the density-functional perturbation theory (DFPT). The predictive power of the DFT/DFPT approach has long been demonstrated through the excellent agreement between theoretical calculations and experimental observations including the recent studies on the lattice vibrational modes of graphite and graphene. ${ }^{6,12}$

In the present analysis, we take advantage of the PWSCF package of the QUANTUM-ESPRESSO distribution ${ }^{13}$ to model the characteristic graphene lattice dynamics. Specifically, an ultrasoft pseudopotential in the local-density approximation $^{14}$ is used with a plane-wave expansion up to 55 Ry and $32 \times 32 \times 1$ Monkhorst-Pack meshes adopted for Brillouin-zone sampling with 0.02 Ry Fermi-Dirac smearing in the electronic occupation. The calculation also employs the theoretical lattice parameter $a=2.46 \AA$, which is in excellent agreement with the experimental value at $300 \mathrm{~K}$. For bilayer graphene, the Bernal stacking is considered with the interlayer distance set to the theoretical minimum of 3.36 Å. 15

Figure 1 provides the calculated phonon dispersion along the high-symmetry directions for (a) monolayer and (b) bilayer graphenes (with the typical $A-B$ stacking). Compared to the available experimental and theoretical data in the literature, ${ }^{6,16-18}$ they are in excellent agreement as summa- 


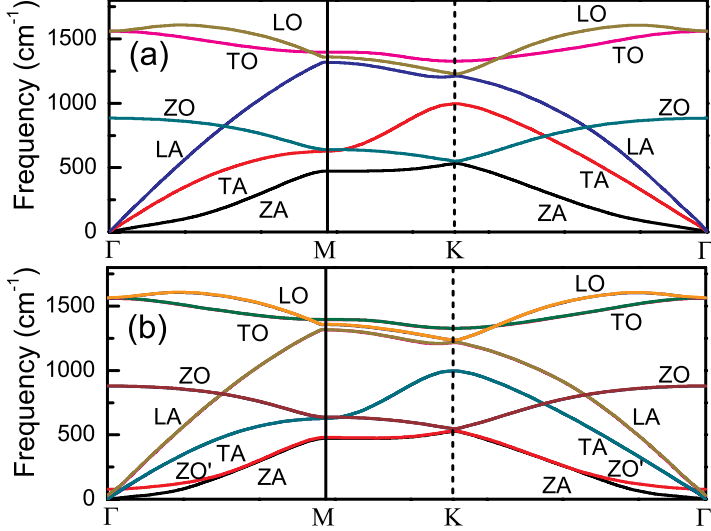

FIG. 1. (Color online) Phonon dispersion of (a) monolayer and (b) bilayer graphenes (with $A-B$ stacking) along the high-symmetry directions. Following the convention, the symbol $\mathrm{Z}$ denotes the outof-plane vibration.

rized in Table I for the monolayer case. Our calculation also seems to support the Kohn-like anomaly in the dispersion of the highest optical branches at the $\Gamma$ and $K$ points. ${ }^{19,20}$ As for bilayer graphene, the phonon band structure exhibits a very similar behavior except the so-called layer-breathing mode (denoted as $\mathrm{ZO}^{\prime}$ ) that gives a nonzero frequency in the longwavelength limit $\left(\Gamma_{\mathrm{ZO}^{\prime}}=78 \mathrm{~cm}^{-1}\right)$. This occurs when the $c$-directional vibration of carbon atoms in adjacent 2D layers becomes out of phase (i.e., optical mode). In graphite the corresponding mode has a frequency of $95 \mathrm{~cm}^{-1} .{ }^{16}$ All other branches (namely, those except $\mathrm{ZO}^{\prime}$ and $\mathrm{ZA}$ ) for bilayer are doubly degenerate with only small splittings that arise from the weak interlayer coupling. The phonon group velocity $\mathbf{v}_{\lambda}(\mathbf{q})$ can be readily obtained by taking a gradient of the dispersion relation for each phonon mode $\lambda$ (not shown).

Similarly, the Grüneissen parameters can be deduced from the negative logarithmic derivative of the phonon frequency with respect to the volume $\gamma_{\lambda}=-\left[a / 2 \omega_{\lambda}(\mathbf{q})\right]\left[d \omega_{\lambda}(\mathbf{q}) / d a\right],{ }^{6,21}$ where $a$ is the lattice constant. For their relevance to the lattice anharmonic properties, these parameters provide crucial information in phonon relaxation as it will be discussed below. The computed Grüneissen parameters are shown in Fig. 2. In monolayer graphene, the $\mathrm{ZO}$ and the $\mathrm{ZA}$ modes have a negative $\gamma$, between -1.38 and -0.17 for $\mathrm{ZO}$ and between -53 and -1.46 for ZA. The values for the other modes range between 0.16 and 2.76. Physically, a positive $\gamma$ corresponds to a decrease in the phonon frequency as the lattice constant increases and the inverse is true for negative

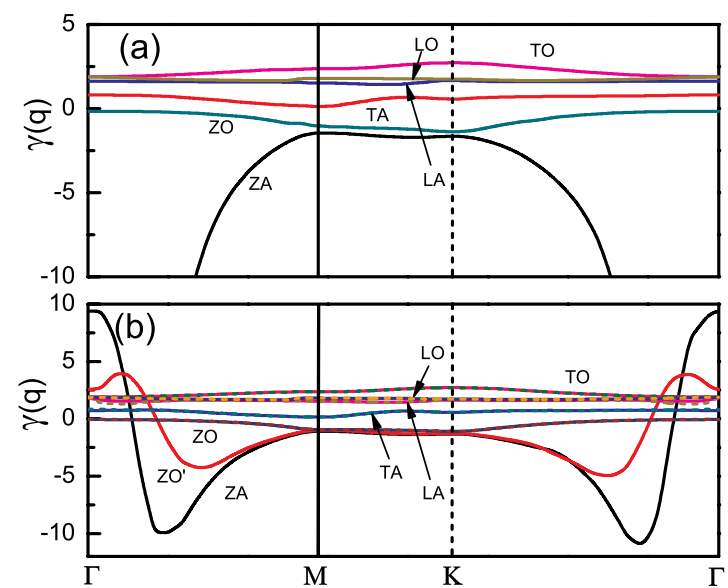

FIG. 2. (Color online) Grüneissen parameter of (a) monolayer and (b) bilayer graphenes (with $A-B$ stacking) along the highsymmetry directions.

$\gamma$. Since ZA and ZO are out-of-plane transverse modes, it is reasonable to expect that the collective vertical motion of the atoms would be more vigorous if a graphene sheet is expanded laterally. As for the bilayer (with $A-B$ staking), the Grüneissen parameters display a different behavior for the $\mathrm{ZA}$ and the $\mathrm{ZO}^{\prime}$ modes near the $\Gamma$ point, where they turn positive. As the volume increases (and, thus, the interlayer distance), the atoms in both layers seem to lose some of their coherence in the long-wavelength limit, leading to a decrease in $\omega_{\mathrm{ZA}}$ and $\omega_{\mathrm{ZO}}$ and the observed positive number for the corresponding $\gamma$. The mean values for the two TA and LA modes in the first Brillouin zone are found to be $0.52\left(\gamma_{\mathrm{TA} 1}\right)$, $0.53\left(\gamma_{\mathrm{TA} 2}\right), 1.58\left(\gamma_{\mathrm{LA} 1}\right)$, and $1.56\left(\gamma_{\mathrm{LA} 2}\right)$, respectively.

The normalized thermal conductivity $\kappa / \tau$ is plotted in Fig. 3. Evidently, the characteristic behaviors of monolayer and bilayer graphenes are much alike over the temperature range of interest $(200-500 \mathrm{~K})$; both show almost the same normalized thermal conductivities. For instance, the combined TA and LA contributions at $T=300 \mathrm{~K}$ give $\kappa / \tau=4.78 \times 10^{13} \mathrm{~W} \mathrm{~m}^{-1} \mathrm{~K}^{-1} \mathrm{~s}^{-1}$ for monolayer and $4.83 \times 10^{13} \mathrm{~W} \mathrm{~m}^{-1} \mathrm{~K}^{-1} \mathrm{~s}^{-1}$ for bilayer. At the same time, the relative significance of the LA modes (dotted line) over the TA phonons (dashed line) is another common feature of the two cases due to the large LA group velocity. This similarity is expected from the essentially identical phonon properties. One interesting point to note is that the magnitude of $\kappa / \tau$ for the individual TA or LA branch in the bilayer is about one half of that in the monolayer. The seeming disparity is due to

TABLE I. Comparison of graphene phonon frequencies at $\Gamma, M$, and $K$ obtained from various studies (in units of $\mathrm{cm}^{-1}$ ).

\begin{tabular}{lcccccccccccccc}
\hline \hline & $\Gamma_{\text {ZO }}$ & $\Gamma_{\mathrm{LO} / \mathrm{TO}}$ & $M_{\mathrm{ZA}}$ & $M_{\mathrm{TA}}$ & $M_{\mathrm{ZO}}$ & $M_{\mathrm{LA}}$ & $M_{\mathrm{LO}}$ & $M_{\mathrm{TO}}$ & $K_{\mathrm{ZA}}$ & $K_{\mathrm{ZO}}$ & $K_{\mathrm{TA}}$ & $K_{\mathrm{LA}}$ & $K_{\mathrm{LO}}$ & $K_{\mathrm{TO}}$ \\
\hline This work & 884 & 1560 & 473 & 627 & 641 & 1318 & 1360 & 1396 & 532 & 550 & 997 & 1210 & 1228 & 1327 \\
Theoretical $^{\mathrm{a}}$ & 881 & 1554 & 471 & 626 & 635 & 1328 & 1340 & 1390 & 535 & 535 & 997 & 1213 & 1213 & 1288 \\
Experimental & $861^{\mathrm{b}}$ & $1590^{\mathrm{b}}$ & $465^{\mathrm{b}}$ & $630^{\mathrm{b}}$ & $670^{\mathrm{b}}$ & $1290^{\mathrm{c}}$ & $1321^{\mathrm{c}}$ & $1389^{\mathrm{d}}$ & $482^{\mathrm{d}}$ & $588^{\mathrm{d}}$ & & $1184^{\mathrm{d}}$ & $1184^{\mathrm{d}}$ & $1313^{\mathrm{c}}$ \\
\hline
\end{tabular}

\footnotetext{
Reference 6 (graphene).

${ }^{b}$ Reference 16 (graphite).

${ }^{\mathrm{c}}$ Reference 17 (graphene).

${ }^{\mathrm{d}}$ Reference 18 (graphite).
} 


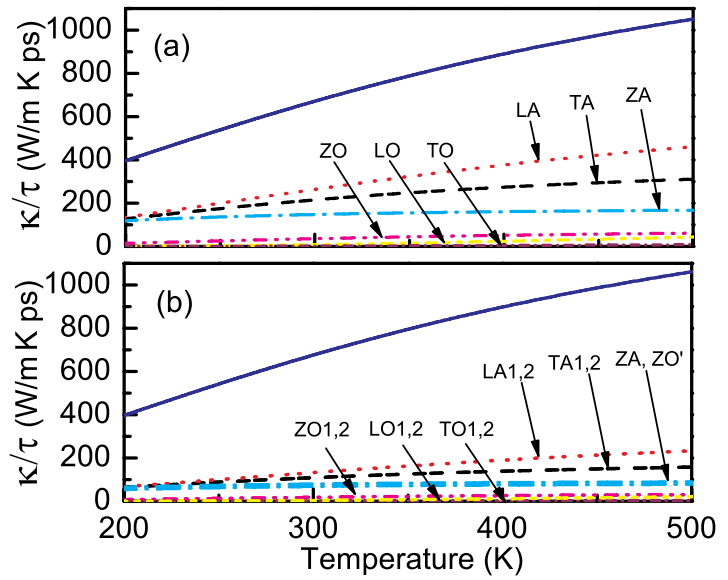

FIG. 3. (Color online) Normalized thermal conductivity $\kappa / \tau$ along the $\Gamma-M$ direction in (a) monolayer and (b) bilayer graphenes with $A-B$ staking. The solid lines denote the combined contribution of all branches. In (b), all of the lines except the solid are doubly degenerate as the contributions of the specified branches essentially overlap.

the fact that the number of phonon modes increases proportionally with the layer thickness as discussed earlier. Accordingly, each branch contributes progressively less while the sum remains approximately the same.

Figure 3 also shows $\kappa / \tau$ of other phonon modes for comparison. As expected, the optical phonons (LO, TO, ZO) provide the numbers that are at least an order-of-magnitude smaller over the entire temperature range. Consequently, the specific features of these branches (such as the Kohn-like anomaly, ${ }^{19,20}$ etc.) have little or no impact on thermal conduction. On the other hand, $\kappa / \tau$ for the $\mathrm{ZA}$ and the $\mathrm{ZO}^{\prime}$ modes becomes non-negligible particularly at low temperatures. A crucial property to note for a detailed analysis is that the dominant contributions come from the phonons away from the zone center (i.e., large wave vectors), as evidenced by nearly degenerate curves of the two branches in bilayer graphene $\left(\mathrm{ZA}\right.$ and $\left.\mathrm{ZO}^{\prime}\right)$ despite the differences in the dispersion relation near the $\Gamma$ point. Since it is the region where the out-of-plane vibrations experience large Grüneissen parameters (see Fig. 2), the corresponding phonons are expected to suffer from rapid decay with a reduced role in thermal transport. This point will be further elaborated below.

To estimate the thermal conductivity $\kappa$ of graphene, we consider the heat conduction primarily via TA and LA modes. Our approach is similar to that of Ref. 2, in which the authors calculated the graphite thermal conductivity along the basal plane considering only the LA and the TA branches. Specifically, this assumption applies to the temperatures above those of the ZA frequency range. With the maximum wave number of the ZA mode around $535 \mathrm{~cm}^{-1}$ (see Table I), it corresponds to the temperatures of approximately $123 \mathrm{~K}$ $\left(k_{B} T=\hbar \omega\right)$. For the phonon lifetime $\tau_{\lambda}$ in Eq. (1), we evaluate it as limited by the anharmonicity of lattice vibrations since this is the most fundamental factor that is not affected by the external conditions such as the purity of crystal or boundary termination processes. An analytical expression for the relaxation rate from the dominant anharmonic three-phonon mechanism can be given for mode $\lambda$ as ${ }^{2,9,22}$

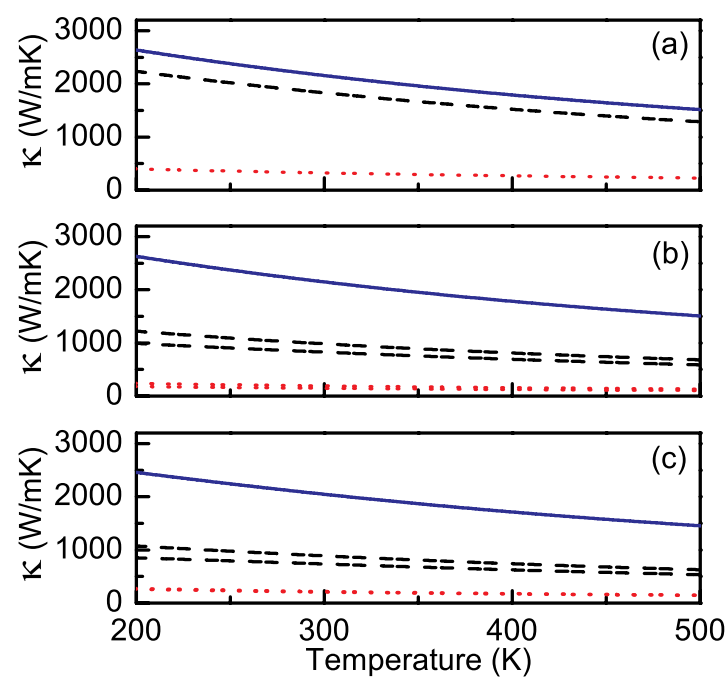

FIG. 4. (Color online) Thermal conductivity $\kappa$ along the $\Gamma-M$ direction in (a) monolayer graphene, (b) bilayer graphene with $A-B$ staking, and (c) bilayer graphene with $A-A$ staking. The dashed and the dotted lines are for the TA and the LA branches, respectively, while the solid lines denote the combined contribution (TA+LA). In (b) and (c), two dotted lines (for two LA modes) essentially overlap with each other and give the false impression of one curve.

$$
\frac{1}{\tau_{\lambda}}=2 \gamma_{\lambda}^{2} \frac{k_{B} T}{M v^{2}} \frac{\omega^{2}}{\omega_{m}},
$$

where $M$ is the atomic mass and $\omega_{m}$ denotes the Debye frequency. For simplicity, the average sound velocity $v$ for a $2 \mathrm{D}$ phonon gas consisting of LA and TA modes is approximated by the relation $2 / v^{2}=1 / v_{\mathrm{LA}}^{2}+1 / v_{\mathrm{TA}}^{2}$ in the long-wavelength limit. As in Ref. 2, the integration in Eq. (1) is truncated with a cutoff frequency of $\Gamma_{\mathrm{ZO}^{\prime}}$ to avoid the divergence issue at the zone center.

Figure 4 presents the lattice thermal conductivity calculated along the $\Gamma-M$ direction. Both monolayer and bilayer graphenes display similar results including the approximate $T^{-1}$ dependence at high temperature. An important point to note here is that the contribution of the TA branch is now much more pronounced than that of the LA branch. This is in contrast to the case of normalized thermal conductivity discussed in Fig. 3. The main reason for the difference between $\kappa$ and $\kappa / \tau$ is a much longer lifetime of TA phonons that can be expected from the comparison of the Grüneissen parameters $\left(\gamma_{\mathrm{LA}} / \gamma_{\mathrm{TA}} \approx 3\right)$. We also examine the angular dependence of the thermal conduction and note only a negligible variation (less than $0.1 \%$ at $300 \mathrm{~K}$ ) for different directions. Accordingly, it shows that the thermal conductivity of the studied graphene layers is nearly isotropic.

As for the specific results, $\kappa$ of approximately $2200 \mathrm{~W} \mathrm{~m}^{-1} \mathrm{~K}^{-1}$ is obtained for monolayer graphene at 300 $\mathrm{K}$. The corresponding value for bilayer graphene [with $A-B$ stacking in Fig. 4(b)] is also very close to the monolayer data with an insignificant deviation on the order of $10 \mathrm{~W} \mathrm{~m}^{-1} \mathrm{~K}^{-1}$. These estimates are in good agreement with Klemens et al. $^{2}$ that predicted $1900 \mathrm{~W} \mathrm{~m}^{-1} \mathrm{~K}^{-1}$ for bulk graphite along the basal plane based on a simple model. The 
difference of approximately $300 \mathrm{~W} \mathrm{~m}^{-1} \mathrm{~K}^{-1}$ is remarkably small considering the differing levels of treatment and materials. More fundamentally, it may be an indication that the number of carbon layers along the $c$ direction has only a minor influence on the in-plane thermal conductivity. To further examine the latter point, we analyze bilayer graphene with a different vertical packing as illustrated in Fig. 4(c). Clearly, changing the staking sequence to $A-A$ does not affect the qualitative characteristics of the estimated lattice thermal conductivity; only the magnitude is reduced slightly (by about $100 \mathrm{~W} \mathrm{~m}^{-1} \mathrm{~K}^{-1}$ at $T=300 \mathrm{~K}$ ) illustrating the insensitivity of in-plane lattice vibrations on the weak interlayer dynamics. Concerning the contributions of flexural/layerbreathing modes, the evaluation of their impact on $\kappa$ is difficult although it is generally believed to be small. ${ }^{2,5}$ If the lifetime model of Eq. (3) is simply applied to the ZA phonons, the calculation suggests additional $45-75 \mathrm{~W} \mathrm{~m}^{-1} \mathrm{~K}^{-1}$ at $300 \mathrm{~K}\left(50-95 \mathrm{~W} \mathrm{~m}^{-1} \mathrm{~K}^{-1}\right.$ at $\left.200 \mathrm{~K}\right)$ to the cases considered above (with the maximum deviation observed in $A-B$ bilayer graphene). This could serve as a measure for the (in)significance of out-of-plane branches with large Grüneissen parameters.

Note that an accurate analysis of the $\mathrm{ZA} / \mathrm{ZO}^{\prime}$ contribution may require a treatment more advanced than Eq. (3) since the latter was developed originally for acoustic phonons under a linear assumption. In fact, even for the TA and the LA modes, this expression [Eq. (3)] or its generalized form ${ }^{5,9,22}$ is expected to provide only an approximate estimate on $\tau$ (and thus, on $\kappa$ ). ${ }^{22}$ As such, it is likely that the discrepancy with the experimental data ${ }^{4}$ is caused, at least in part, by the limited precision of the model. Although these issues may be resolved by an $a b$ initio approach that considers phononrelaxation processes in the frame work of first-principles calculation via the third-order anharmonic terms, its implementation has not yet been reported in the literature. When the problem comes to a realistic finite graphene sheet/ribbon, other relaxation mechanisms can also arise via, in particular, the scattering with impurities/defects, discontinuous ribbon edge states, surface roughness, etc. The impacts of such factors are sample dependent and difficult to gauge accurately due to the lack of relevant information at the moment. Accordingly, our calculation for the intrinsic lattice thermal conductivity should be regarded as a rough estimate while the normalized value $\kappa / \tau$ is expected to be robust.

This work was supported, in part, by the NERC/NIST SWAN-NRI and the DARPA/HRL CERA programs. M.B.N. wishes to acknowledge partial support from the Office of Basic Energy Sciences, U.S. Department of Energy at Oak Ridge National Laboratory under Contract No. DE-AC0500OR22725 with UT-Battelle, LLC. *kwk@ncsu.edu

${ }^{1}$ A. K. Geim and K. S. Novoselov, Nature Mater. 6, 183 (2007).

${ }^{2}$ P. G. Klemens and D. F. Pedraza, Carbon 32, 735 (1994).

${ }^{3}$ A. A. Balandin, S. Chosh, W. Bao, I. Calizo, D. Teweldebrhan, F. Miao, and C. N. Lau, Nano Lett. 8, 902 (2008).

${ }^{4}$ S. Ghosh, I. Calizo, D. Teweldebrhan, E. P. Pokatilov, D. L. Nika, A. A. Balandin, W. Bao, F. Miao, and C. N. Lau, Appl. Phys. Lett. 92, 151911 (2008).

${ }^{5}$ D. L. Nika, E. P. Pokatilov, A. S. Askerov, and A. A. Balandin, Phys. Rev. B 79, 155413 (2009).

${ }^{6}$ N. Mounet and N. Marzari, Phys. Rev. B 71, 205214 (2005).

${ }^{7}$ R. M. Martin, Phys. Rev. B 1, 4005 (1970).

${ }^{8}$ R. Berman, P. R. W. Hudson, and M. Martinez, J. Phys. C 8, L430 (1975).

${ }^{9}$ P. G. Klemens, in Thermal Conductivity, edited by R. P. Tye (Academic, London, 1969), Vol. 1, p. 1.

${ }^{10}$ J. M. Ziman, Electrons and Phonons (Oxford University Press, Oxford, 1960), p. 306.

${ }^{11}$ A. A. Kiselev, K. W. Kim, and M. A. Stroscio, Phys. Rev. B 62, 6896 (2000).

${ }^{12}$ N. Bonini, M. Lazzeri, N. Marzari, and F. Mauri, Phys. Rev. Lett. 99, 176802 (2007).

${ }^{13}$ QUANTUM-ESPRESSO is a community project for high-quality quantum-simulation software based on the DFT and coordinated by Paolo Giannozzi. See http://www.quantum-espresso.org and http://www.pwscf.org

${ }^{14}$ C.pbe.rrjkus.UPF in the standard QUANTUM ESPRESSO distribution from http://www.quantum-espresso.org

${ }^{15}$ The layer thickness of $h=3.5 \AA$ is assumed for monolayer and $h=3.5+3.36=6.86 \AA$ for bilayer. See also A. Gupta, G. Chen, P. Joshi, S. Tadigadapa, and P. C. Eklund, Nano Lett. 6, 2667 (2006).

${ }^{16}$ C. Oshima, T. Aizwa, R. Souda, Y. Ishizawa, and Y. Sumiyoshi, Solid State Commun. 65, 1601 (1988).

${ }^{17}$ J. Maultzsch, S. Reich, C. Thomsen, H. Requardt, and P. Ordejón, Phys. Rev. Lett. 92, 075501 (2004).

${ }^{18}$ H. Yanagisawa, T. Tanaka, Y. Ishida, M. Matsue, E. Rokuta, S. Otani, and C. Oshima, Surf. Interface Anal. 37, 133 (2005)

${ }^{19}$ S. Piscanec, M. Lazzeri, F. Mauri, A. C. Ferrari, and J. Robertson, Phys. Rev. Lett. 93, 185503 (2004).

${ }^{20}$ M. Mohr, J. Maultzsch, E. Dobardzić, S. Reich, I. Milosević, M. Damnjanović, A. Bosak, M. Krisch, and C. Thomsen, Phys. Rev. B 76, 035439 (2007).

${ }^{21}$ N. W. Ashcroft and N. D. Mermin, Solid State Physics (Holt, Rinehart and Winston, New York, 1976), Chaps. 1 and 25.

${ }^{22}$ P. G. Klemens, in Solid State Physics, edited by F. Seitz and D. Turnbull (Academic, New York, 1958), Vol. 7, p. 1. 